\title{
EVALUACIÓN NUTRICIONAL EN UN GRUPO DE ADOLESCENTES EMBARAZADAS EN CARTAGENA, COLOMBIA. ESTUDIO DE CORTE TRANSVERSAL
}

\section{A cross sectional study of nutrition in a group of pregnant adolescents firom Cartagena, Colombia}

Edgar Rivas-Perdomo, M.D.*, Ruth Álvarez, M.D**, Gloria Mejía***

Recibido: septiembre 29/2005 - Revisado: noviembre 29/2005 - Aceptado: diciembre 16/2005

\section{RESUMEN}

Objetivo: evaluar el estado nutricional de un grupo de adolescentes embarazadas en la ciudad de Cartagena, Colombia.

Materiales y Métodos: estudio de corte transversal. Se escogieron 25 adolescentes embarazadas de una población de 40 que reciben apoyo de una fundación en Cartagena, Colombia. Se midieron variables antropométricas y bioquímicas.

Resultados: se encontró un predominio de bajo peso preconcepcional con promedios por debajo del percentil 5 para la edad, reflejados en el índice de masa corporal. Se reportaron valores de hemoglobina y hematocrito tan bajos como de $7 \mathrm{~g} / \mathrm{dl}$ y $20 \%$ respectivamente. También se encontró déficit de linfocitos en 50\% de las mismas. Al aplicar el nomograma de Rosso-Mardones sólo el 24\% de las pacientes fue normal.

Conclusiones: se identificó una deficiencia nutricional en las adolescentes embarazadas. Hay

* Residente de Ginecología y Obstetricia. Universidad de Cartagena Avenida Cartagena $\mathrm{N}^{\circ}$ 32-43. Cartagena, Colombia. Correo electrónico: edrivas@hotmail.com

** Nutricionista y Dietista, Universidad del Atlántico

*** Bacterióloga. Clínica Madre Bernarda, Cartagena. Docente Facultad de Bacteriología Universidad de San Buenaventura, Cartagena. una inadecuada ingesta calórica con valores entre 594-2150 Kcal./día.

Palabras clave: nutrición, índice de masa corporal, ingesta de dieta, embarazo y adolescencia.

\section{SUMMARY}

Objective: the purpose of this study was to evaluate the nutritional state of a group of pregnant adolescents from Cartagena, Colombia.

Study design: 25 pregnant adolescents were chosen from a population of 40 receiving help from a foundation in Cartagena, Colombia. Anthropometric and biochemical variables were measured.

Results: low pre-pregnancy maternal weight was prevalent for this age group, means lying below the $5^{\text {th }}$ percentile as reflected in the body mass index. Very low $7 \mathrm{~g} / \mathrm{dl}$ haemoglobin and 20\% haematocrite values were presented and 50\% lymphocyte deficit revealed in these patients. The Rosso-Mardones nomogram was normal in just 24\% of them.

Conclusion: there was inadequate calorie intake in the population being studied, values ranging from 594-2,150 Kcal/day. Nutritional deficiency was thus identified in this particular group of pregnant adolescents.

Key words: nutrition, body mass index, dietary intake, pregnancy during adolescence. 


\section{INTRODUCCIÓN}

El hombre, como ser vivo es termodinámicamente inestable, por lo que necesita un suministro continuo de energía que le va a ser proporcionada por los alimentos. ${ }^{1}$ Esta condición es particularmente significativa durante el embarazo, cuando se producen numerosos cambios profundos en el metabolismo materno que sirven para proporcionar sustratos adecuados para el crecimiento y desarrollo del feto. ${ }^{2}$ El costo calórico acumulativo del embarazo se ha calculado en aproximadamente $85.000 \mathrm{Kcal}$ más de la ingestión usual. ${ }^{3}$ El Instituto de Medicina de las Academias Nacionales de Estados Unidos (Institute of Medicine) en 1990 estima los requerimientos totales de energía durante el embarazo ${ }^{4} \mathrm{y}$ constituye la base de la recomendación actual de que la embarazada consuma $300 \mathrm{Kcal}$ adicionales por día durante el segundo y tercer trimestres. ${ }^{5,6}$ La adolescente embarazada (5 años postmenarquia) debe ganar entre $14-15 \mathrm{Kg}$. a una velocidad de $500 \mathrm{~g} / \mathrm{semana}$. Esta paciente debe ganar casi 4 gr por cada gramo de peso fetal debido a las necesidades de su propio crecimiento. ${ }^{3}$ Durante el embarazo, la proteína adicional se requiere para el desarrollo fetal y placentario y extensión de los tejidos maternos. ${ }^{7}$ Es necesaria una ingesta de proteínas incrementada a 60 gr/día, un incremento inadecuado de proteínas lleva a anemia materna y restricción del crecimiento fetal. ${ }^{8}$ Estos requerimientos se ven aumentados en la adolescencia por las razones expuestas anteriormente. ${ }^{9}$

Estos cuidados alimentarios son más importantes en las adolescentes embarazadas ya que deben sumar a las necesidades propias del embarazo, las originadas por no haber completado su desarrollo y el feto en desarrollo puede competir con la adolescente en crecimiento. ${ }^{3,4}$ El factor de riesgo nutricional materno es elevado en países en desarrollo. En Chile, en 334 madres con recién nacidos a término y sin patologías, solo el 54\% presentaba características nutricionales previas, definidas como normales, ${ }^{10}$ contribuyendo significativamente a los altos índices de riesgo obstétrico.
El embarazo en adolescentes constituye una gran problemática social tanto en países industrializados como en aquellos llamados en vías de desarrollo, lo cual a juzgar por los datos reportados constituyen un problema de salud pública. Se ha reportado una asociación entre un índice de masa corporal muy bajo o muy alto antes del embarazo y malos resultados perinatales tales como prematuridad y restricción del crecimiento intrauterino ${ }^{11}$ incluyendo desnutrición y muerte fetal intrauterina, aunque esto último ha sido controversial. ${ }^{12,13,3}$

En Colombia como en todos los países no industrializados es común que las mujeres tengan su primer hijo en la adolescencia, sobre todo en los sectores de la población mas deprimidos social, económica y culturalmente y en las cuales ya pueden existir deficiencias nutricionales. ${ }^{14}$ No obstante la importancia referida a la nutrición en la embarazada, poco se conoce sobre los cambios y adaptaciones fisiológicas y nutricionales que ocurren en ellas; la mayoría de los estudios describen el comportamiento sicosocial de la adolescente embarazada. Este estudio pretende hacer una aproximación a la frecuencia de desnutrición en las adolescentes embarazadas en nuestra región utilizando variables antropométricas de fácil medición como el peso y talla que facilitan la determinación del índice de masa corporal (IMC); la determinación de la reserva muscular y parámetros bioquímicos como hemograma, leucograma y proteinograma, así como el índice de creatinina-talla. Igualmente se pretende establecer los hábitos alimenticios y con ello establecer el riesgo de problemas nutricionales en la población objeto del estudio.

\section{MATERIALES Y MÉTODOS Recolección de la información}

Diseño: estudio de corte transversal.

Población y lugar: 25 adolescentes embarazadas de un grupo de 40 que reciben apoyo de la Fundación "Juan Felipe Gómez Escobar"; provenientes de diversos sectores de la ciudad que admitieron voluntariamente ingresar al grupo de estudio y 
permitieron la obtención de las muestras de sangre y orina en 24 horas. El estudio se realizó en la ciudad de Cartagena, durante los meses de marzo y abril de 2005, previa aprobación por las directivas de la fundación.

Tamaño muestral: 25 pacientes

Procedimiento: se realizó una encuesta evaluando el estado nutricional mediante medidas de variables antropométricas, hematológicas y bioquímicas. Se evaluó el hábito alimenticio utilizando la técnica de recordatorio en 24 horas y la tabla de conversión propuesta por la Facultad de Nutrición y Dietética de la Pontificia Universidad Javeriana. ${ }^{15}$

\section{Variables a medir}

Antropométricas: edad, peso y talla antes de la gestación. Se determinó el índice de masa corporal (IMC) pregestacional con base en la talla y peso antes del embarazo, y su ubicación en el percentil para el mismo de acuerdo a la edad de la paciente. Sólo fue posible obtener el valor del IMC en 18 pacientes quienes recordaban su peso antes de la gestación. Se determinó el área muscular del brazo, para lo cual fue necesario medir la circunferencia del brazo y el espesor del pliegue del tríceps (EPT) y aplicar la fórmula: = Circunferencia del brazo - 0,314 x (EPT). y el índice Peso-Talla aplicando el nomograma propuesto por Rosso- Mardones en 1987, según Barrera. ${ }^{16}$

Valoración hematológica y bioquímica: recuento de leucocitos y linfocitos en sangre periférica, concentraciones de hemoglobina $(\mathrm{Hb})$ y hematocrito por método automatizado con un Advia-60. Albúmina sérica y creatinina en orina de 24 horas con espectrofotómetro RA-50. Con la creatinina en orina de 24 horas se determinó el índice creatinina/talla. La anemia materna, es definida por un hematocrito menor de 32\% y niveles de hemoglobina menores de $11 \mathrm{~g} / \mathrm{dl}$. Todos los parámetros bioquímicos se interpretaron de acuerdo con las escalas de valores citados por Vargas. ${ }^{17}$

\section{ANÁLISIS}

Se realizó una base de datos mediante el software Epi-Info 2004. Versión 3.2. Las variables continuas se describen por medio de medidas de tendencia central y dispersión, las variables categóricas por medio de proporciones.

\section{RESULTADOS}

En la tabla 1 se presentan las características demográficas de la población estudiada. La edad gestacional promedio de las adolescentes estudiadas fue de 25,9 土 7,5 semanas. El 88,2\% de las mismas eran primigestantes. Se encuentra que el 8\% de las adolescentes estudiadas, estaban viviendo con su pareja, con una pobre escolaridad e importante deserción escolar, donde sólo cuatro continúan estudiando.

Sólo en 18 de ellas se obtuvo el valor del IMC pregestacional. Y se ubicaron en el percentil $10 \mathrm{el}$ 39\% de las adolescentes. 3 jóvenes se ubicaron por debajo del percentil 5. (Los valores de percentiles

\begin{tabular}{|c|c|c|c|}
\hline Estado civil & $\mathbf{N}$ & $\%$ & $\begin{array}{c}\% \\
\text { acumulado }\end{array}$ \\
\hline Soltera & 13 & 52 & 52 \\
\hline Unión libre & 12 & 48 & 100 \\
\hline Total & 25 & 100 & 100 \\
\hline \multicolumn{4}{|l|}{ Escolaridad } \\
\hline Primaria completa & 2 & 8,0 & 8,0 \\
\hline Primaria incompleta & 3 & 12,0 & 20,0 \\
\hline Secundaria completa & 4 & 16,0 & 36,0 \\
\hline Secundaria incompeta & 16 & 64,0 & 100,0 \\
\hline Total & 25 & 100,0 & 100,0 \\
\hline \multicolumn{4}{|l|}{ Ocupaciones } \\
\hline Ama de casa & 14 & 56,0 & 56,0 \\
\hline Actividad de belleza & 2 & 8,0 & 64,0 \\
\hline Desempleada & 4 & 16,0 & 80,0 \\
\hline Estudiante & 4 & 16,0 & 96,0 \\
\hline Ventas ambulantes & 1 & 4,0 & 100,0 \\
\hline Total & 25 & 100,0 & 100,0 \\
\hline
\end{tabular}

Fuente: Fundación Juan Felipe Gómez Escobar. Cartagena 
Tabla 2. Valores antropométricos de un grupo de adolescentes embarazadas en Cartagena

\begin{tabular}{|c|c|c|c|c|c|c|c|c|c|c|c|c|}
\hline \multirow{2}{*}{ Edad (años) } & \multirow{2}{*}{$\mathrm{N}$} & \multirow{2}{*}{$\%$} & \multirow{2}{*}{$\begin{array}{c}\text { Peso } \\
\text { (kg.) } \bar{X}\end{array}$} & \multirow{2}{*}{ Percentil } & \multirow{2}{*}{$\begin{array}{c}\text { Talla } \\
\text { (cm.) } \bar{X}\end{array}$} & \multirow{2}{*}{ Percentil } & \multicolumn{6}{|c|}{ IMC percentiles } \\
\hline & & & & & & & $\leq 5$ & 10 & 25 & 50 & 75 & $>75$ \\
\hline 14 & 1 & 4 & - & - & 158 & 40 & - & - & - & - & - & - \\
\hline 15 & 4 & 16 & 41,8 & $<5$ & 152,5 & 75 & - & 1 & 1 & 1 & & 1 \\
\hline 16 & 11 & 44 & 42,8 & $<5$ & 154,4 & $>75$ & 3 & 1 & - & 2 & 1 & - \\
\hline 17 & 4 & 16 & 39,6 & $<5$ & 147,7 & 70 & - & 1 & 2 & - & - & \\
\hline 18 & 5 & 20 & 46,0 & 7 & 156 & $>75$ & - & 2 & 2 & - & - & - \\
\hline
\end{tabular}

$\bar{X}=$ Valor promedio

Fuente: Fundación Juan Felipe Gómez Escobar. Cartagena

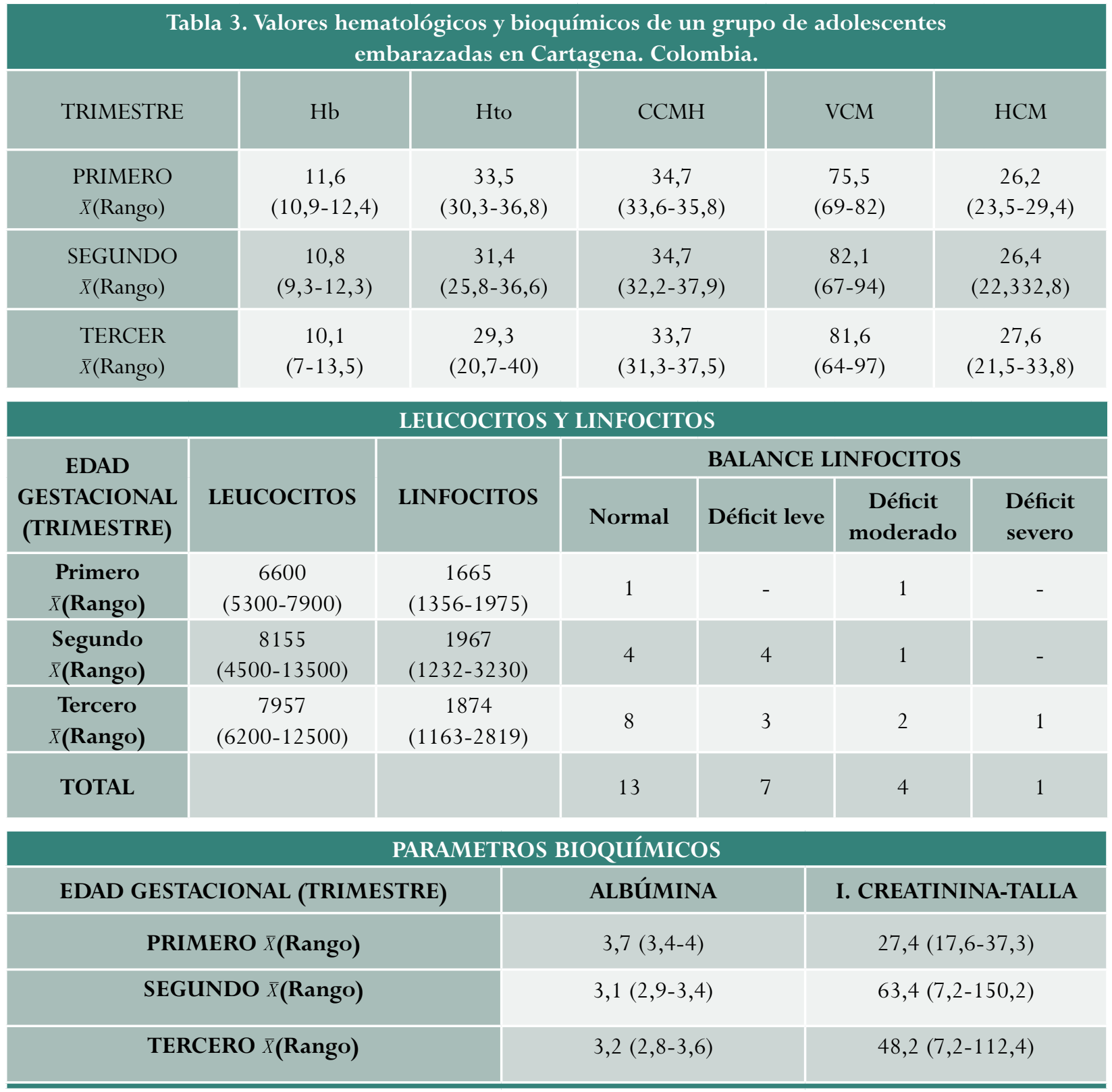

$\bar{X}$ : Valor promedio; Rango: Límite de los valores encontrados

Fuente: Fundación Juan Felipe Gómez Escobar. Cartagena 


\begin{tabular}{|c|c|c|c|}
\hline EDAD GESTACIONAL (TRIMESTRE) & NORMAL & BAJO PESO & SOBREPESO \\
\hline PRIMERO & - & 2 & - \\
\hline SEGUNDO & 6 & 2 & 1 \\
\hline TERCERO & - & 13 & 1 \\
\hline TOTAL & 6 & 17 & 2 \\
\hline
\end{tabular}

Fuente: Fundación Juan Felipe Gómez Escobar. Cartagena

de estas 18 jóvenes se detallan en la tabla 2. La circunferencia del brazo en promedio fue de 24,8 cm. con una desviación estándar $(\mathrm{DE})=5,9$ en un rango entre 20 y 49 . El pliegue del tríceps tuvo un valor promedio de $12,2 \mathrm{~mm}$ con una $\mathrm{DE}=4,86$ en un rango entre 6,0 y 30,0 mm. El área muscular fue en promedio de 29,3 $\mathrm{cm}^{2}$ con una $\mathrm{DE}=14,5$ en un rango entre 13,0 y 84,5 . Sólo una joven tuvo un área muscular de $13,0 \mathrm{~cm}^{2}$.

Los valores hematológicos y los bioquímicos se presentan en las tabla 3. Al aplicar el nomograma propuesto por Rosso-Mardones, se encuentra que sólo el 24\% se ubican dentro del rango de la normalidad, mientras que el 68\% están en bajo peso y el $8 \%$ en sobrepeso. (Tabla 4).

La ingesta de alimentos tuvo un promedio de 1486,7 Kcal con una DE de 412,70 en un rango entre 594 y $2150 \mathrm{Kcal} /$ día. En el 38,8\% de las adolescentes encuestadas se encontró una ingesta < $1500 \mathrm{Kcal} /$ día el 50\% entre 1500-2000 y el 11,1\% $>2000 \mathrm{Kcal} /$ día. La dieta estuvo representada en 54,6 + 9,9\% por glúcidos (rango: 39-74\%), lo que equivalía a un promedio de 201,6 \pm 56,0 gr en un rango entre 60-293 gr. Proteínas: 15,8 \pm 2,8\% (Rango: 10-20\%) equivalente a 60,1 $+24,1$ gr en un rango entre 21-108,5 gr. Grasas: 39,6 \pm 9,0\% (Rango: 9-4\%), equivalente a 48,8 \pm 19,7 gr en un rango entre 10-82,5 gr. La relación de grasas saturadas / insaturadas tuvo un valor promedio de 1,043 $\pm 0,524$, en un rango entre 0,35 y 2,07 .

\section{DISCUSION Y CONCLUISIONES}

En nuestra serie la edad promedio de las pacientes se encuentra por encima de los valores reportados por Casanueva ${ }^{18}$ quien reporta valores promedio de 15 años con un rango entre 11 y 17 años. Rivas y Cermeño ${ }^{19}$ encontraron que las adolescentes de una comunidad estudiada iniciaban vida sexual activa en promedio a los 15,1 años y vida obstétrica a los 15,5 años. La alta deserción escolar es el reflejo de la magnitud del problema que constituye el embarazo en las adolescentes y que ha llevado a algunos autores a considerarlo un problema de salud pública. Son jóvenes que sin tener un adecuado desarrollo orgánico ni mental deben enfrentar un proceso de ajuste de su identidad, y según $\mathrm{Kano}^{20}$ asumen el rol de adultos. La edad gestacional coincide con lo encontrado por Casanueva. ${ }^{18}$

Las medidas antropométricas sirven para establecer el estado del compartimiento calórico. ${ }^{3} \mathrm{Se}$ encontraron valores próximos a los reportados por Barrera $^{16}$ quien encontró una circunferencia del brazo de 27,1 cm DE 2,5; un pliegue del tríceps de 16,5 \pm 4,8 mm, y un área muscular de 37,9 DE: 8,1 en una población adulta en una región del interior del país. Según Hamaoui ${ }^{3}$ la medida precisa del espesor de la piel poco aporta a esta valoración durante el embarazo. Sin embargo, el área muscular del brazo es tenido como un indicador del compartimiento muscular esquelético y del comportamiento proteico corporal, cuyo valor promedio establecido es $23,2 \mathrm{~cm}$.

El predominio de bajo peso preconcepcional con promedios por debajo del percentil 5 para la edad, que se refleja en el índice de masa corporal $^{3}$ indicaría una significativa desnutrición en la adolescente antes del embarazo. Estos resultados contrastan con los reportados por Reina ${ }^{14}$ 
quien no encontró desnutrición en adolescentes de Cali (Colombia). Los valores de hemoglobina y hematocrito se encuentran en una proporción menor que la reportada por Casanueva, ${ }^{18}$ quien encuentra que 8 de cada 10 adolescentes tiene anemia y deficiencia de hierro. En nuestra serie la proporción equivale a un 4,4\%. Sin embargo se encuentra una frecuencia mayor que la reportada por Levario - Carrillo ${ }^{21}$ en una población general del 17\% y Marín ${ }^{22}$ del 16\%. Los índices eritrocitarios promedios de volumen corpuscular medio (VCM) y hemoglobina corpuscular media (HCM) se registraron por debajo de los valores normales lo cual corresponde a microcitosis y que está en relación con la anemia ferropénica, que en los embarazos de adolescentes se relacionan con depleción de hierro. ${ }^{23}$ Sin embargo, los valores de la concentración de hemoglobina corpuscular media (CCMH) que se considera un índice mas confiable durante el embarazo, en ningún caso fue inferior del 30\% que puede ser explicado por el hecho de que en un comienzo las anemia ferroprivas son normocíticas, normocrómicas. ${ }^{23}$ Estos hallazgos se correlacionan con los reportados por Agudelo ${ }^{24}$ en una población de Medellín. Nuestra serie presenta un número pequeño de pacientes lo que conlleva a la amplia variabilidad de las observaciones.

La desnutrición proteico calórica es una de las causas mas comunes de inmunodeficiencia adquirida, así que si hay depleción proteica, lo mas seguro es que el recuento de linfocitos esté disminuido, lo cual explica los bajos valores obtenidos. ${ }^{16}$ La albúmina es un indicador de depleción tisular y la hipoalbuminemia se relaciona con una síntesis disminuida, degradación aumentada, pérdidas aumentadas o cambios en la composición y cantidad de líquidos corporales. Según Vargas, ${ }^{17}$ la hipoalbuminemia por destrucción es de aparición tardía, ello podría explicar la normalidad de los valores en las pacientes del primer trimestre y no así en los subsecuentes. En general, los parámetros bioquímicos sirven para establecer el estado del compartimiento proteico y ello se ha visto disminuido en un alto porcentaje de las adolescentes estudiadas. La ingesta calórica ideal: esto está lejos de ser alcanzado con ingesta tan pobres como las que registramos en este estudio cuando sus propias necesidades de crecimiento compiten con las del feto, en razón de que estas aún no han completado su desarrollo.

Se identificó una deficiencia nutricional en las adolescentes embarazadas objeto de estudio. Hay una inadecuada ingesta calórica con valores entre 594-2150 Kcal./día. Pero por el tamaño de la muestra no es posible extrapolar los resultados a la población de Cartagena, por lo cual se hace necesario la realización de un estudio con mas pacientes y en un diseño de cohorte que siga a las pacientes hasta por lo menos un año de vida del recién nacido.

\section{REFERENCIAS}

1. Varela G. Nutrición. En: Tresguerres JAF. Fisiología humana. Madrid: Interamericana McGraw-Hill; 1992.

2. Resnik R, Brace RA. Fisiología del embarazo. En. West JB. Best y Taylor. Bases fisiológicas de la práctica médica. 12 ed. Buenos Aires: Edit. Médica panamericana; 1993.

3. Hamaoui E, Hamaoui M. Nutritional assessment and support during pregnancy. Gastroenterol Clin North Am 2003;32:59-121.

4. Reifsnider E, Gill SL. Nutrition for the childbearingyears. J Obstet Gynecol Neonatal Nurs 2000;29:43-55.

5. Abrams B. Aumento de peso e ingestion energética durante el embarazo. Clin Obstet Ginecol 1994;3:473-84.

6. Abrams B, Pickett KE. Maternal nutrition. En: Creasy RK, Resnik R. Maternal-fetal medicine. 4 ed. Philadelphia: W.B. Saunders Company; 1999.

7. James DC. Eating disorders, fertility, and pregnancy: relationships and complications. J Perinat Neonatal Nursing 2001;15:36-48.

8. Fowles ER. Comparing pregnant women's nutritional knowledge to their actual dietary intake. Am J Matern Child Nurs 2002;27:171-7.

9. González de Agüero R, Fabre E, Sobreviela M, Morollon I. Recomendaciones dietéticas durante el embarazo y la lactancia. En: González de Agüero R, Fabre E. Nutrición y dietética durante el embarazo. Barcelona: Masson; 1996. 
10. Donoso E. Nutrición materna y embarazo. En: Oyarzún E. Alto riesgo obstétrico. Santiago, Chile: Universidad Católica de Chile; 1998. p. 27-38.

11. Ehrenberg M, Dierker L, Milluzzi C; Mercer BM. Low maternal weight, failure to thrive in pregnancy, and adverse pregnancy outcomes. Am J Obstet Gynecol 2003;189:1726-30.

12. Stephansson O, Dickman PW, Johansson A, Cnattingius S. Maternal weight, pregnancy weight gain, and the risk of antepartum stillbirth. Am J Obstet Gynecol 2001;184:463-9.

13. Felice ME, Feinstein RA, Fisher MM, Kaplan DW, Olmedo LF, Rome ES, et al. Adolescent pregnancy --current trends and issues: 1998 American Academy of Pediatrics Committee on Adolescence, 1998-1999. Pediatrics 1999;103:516-20.

14. Reina JC, Orozco B, Dufor D, Spurr G. Adolescentes y embarazo: salud y nutrición. Rev Colomb Obstet Ginecol 2000;51:195-217.

15. Díaz M. XIV Congreso Anual Avances en Metabolismo y Soporte Nutricional. Cartagena; 2000.

16. Barrera M, Cárdenas N, Vergara N. Determinación de parámetros antropométricos para la valoración nutricional de la gestante. Rev Fac Med Univ Nal Col 1998;46:16-21.

17. Vargas CR. Valoración nutricional de la paciente embarazada. Salud procreativa y perinatal 1996;2:13-21.
18. Casanueva E, Jimenez J, Meza-Camacho C, Mares M, Simon L. Prevalence of nutritional deficiencies in Mexican adolescent women with early and late prenatal care. Arch Latinoam Nutr 2003;53:35-8.

19. Rivas-Perdomo EE, Cermeño RD. Estudio piloto de caracterización general de la etapa reproductiva en mujeres de Cáceres, Antioquia, Colombia. Controversias en Ginecología y Obstetricia 2001;8:309-19.

20. Kano ME. El proceso de enfrentar un embarazo en la adolescencia: ajustando la identidad. Controversias en Ginecología y Obstetricia 1999;2:14-24.

21. Levario-Carrillo M, Hernandez M, Vasquez ME, Chavez D, Sanchez C, Corral M. [Effects of irondeficiency anemia on placenta and birth weight]. Ginecol Obstet Mex 2003;71:75-81.

22. Marin GH, Fazio P, Rubbo S, Baistrocchi A, Sager G, Gelemur A. [Prevalence of anaemia in pregnancy and analysis of the underlying factors]. Aten Primaria 2002;29:158-63.

23. Elkins SL, Perry KG. Anemia ferropénica. En: Gleicher N. Tratamiento de las complicaciones clínicas del embarazo. 3 ed. Buenos Aires: Panamericana.

24. Agudelo GM, Cardona OL, Posada M, Montoya MN, Ocampo NE, Marin CM, et al. Prevalence of irondeficiency anemia in schoolchildren and adolescents, Medellín, Colombia, 1999. Pan Am J Public Health June 2003 [Visitado 2005 Nov 29]. Disponible en: http://publications.paho.org. 